\title{
Brain organoids: a next step for humanized Alzheimer's disease models?
}

\author{
Yannis Gerakis ${ }^{1,2,3} \cdot$ Claudio Hetz $^{1,2,3,4,5}$
}

Received: 22 October 2018 / Revised: 20 November 2018 / Accepted: 10 December 2018 / Published online: 7 January 2019

(c) Springer Nature Limited 2019

A major and actual issue in the field of neurodegenerative diseases is the poor translational potential of most preclinical models [1]. In the context of Alzheimer's disease (AD), a number of promising pharmaceutical approaches yielded impressive results in various in vitro systems and animal models where they almost cured the disease, but ultimately failed in providing effective protection in clinical trials [2]. Protein misfolding is a hallmark of AD, involving the accumulation of amyloid- $\beta$ peptide $(\mathrm{A} \beta)$ and hyperphosphorylated Tau protein in discrete areas of the brain [3]. These lesions are proposed as a triggering step of a cascade of molecular events resulting in synaptic dysfunction and neuronal degeneration, leading to cognitive and memory impairment. While the vast majority of $\mathrm{AD}$ cases are sporadic, familial cases (FAD) are extremely aggressive with an earlier onset and are usually associated to exacerbated amyloidopathy [4]. Mutations on the genes encoding the amyloid precursor protein (APP) gene or presenilin 1 (PS1), the catalytic component of the $\gamma$-secretase complex that produce the $A \beta$ peptide, are responsible for most of FAD cases. For this reason, widely used mouse models of $\mathrm{AD}$ are genetically engineered to overexpress high levels of the human APP in combination or not with human PS1, where both genes carry one or multiple FAD mutations [5].

Claudio Hetz

chetz@hsph.harvard.edu

chetz@med.uchile.cl

1 Biomedical Neuroscience Institute, Faculty of Medicine, University of Chile, Santiago, Chile

2 Center for Geroscience, Brain Health and Metabolism, Santiago, Chile

3 Cellular and Molecular Biology Program, Institute of Biomedical Sciences, University of Chile, Santiago, Chile

4 Buck Institute for Research on Aging, Novato, CA 94945, USA

5 Department of Immunology and Infectious Diseases, Harvard School of Public Health, Boston, MA, USA
These models produce large amounts of $\mathrm{A} \beta$ peptide, generally resulting in memory impairment at a young age. Transgenic mice have been invaluable in elucidating some of the molecular mechanisms underlying AD pathogenesis but they suffer from several limitations [5]. Among them, a central critic is the difficulty to reproduce Tau pathology $[5,6]$. The expression of APP or PS1 human mutations in $\mathrm{AD}$ transgenic mice is unable to fully recapitulate this central lesion, unless a human Tau transgene is expressed concomitantly [7]. In addition, most trangenic mice develop histopathological AD features early in life, which is a major limitation to study the molecular events priming the development of the pathology during aging [5, 7]. Furthermore, despite that the murine genome contains most AD-related genes, mice do not spontaneously develop an AD phenotype during aging, suggesting that the molecular networks driving disease initiation may not be present in this specie. Thus, alternative systems are needed to study these pathological mechanisms and test the efficacy of new therapeutic strategies. The development of humanized models is emerging as a powerful complementary approach to overcome the limitations of current $\mathrm{AD}$ transgenic mice (see Fig. 1). In a recent issue of Molecular Psychiatry, Claudio Soto's group developed a consistent characterization of brains organoids from cell derived of a human FAD patient, recapitulating the major hallmarks of the disease, including the interplay between $\mathrm{A} \beta$ peptide accumulation and Tau phosphorylation [8].

The technical advances in human somatic cells reprogramming into induced pluripotent stem cells (iPSCs) enabled new research angles to study human disease and organ development [9]. The improvement of in vitro protocols for the differentiation of iPSCs led to the generation of "organoids", which are defined as self-organized and self patterning three-dimensional (3D) structures that share some similarities with complex organs. Brain organoids, also known as mini-brains, recapitulate the developmental process of the brain in the test tube, generating organized structures broadly resembling different regions of the brain 


\section{Features}

'Humanized' animal models

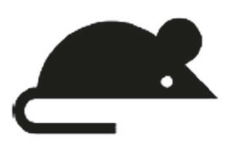

Overexpression / Knock-in human gene APP, PS1

carrying familial mutations

\section{D iPSC neurons culture}

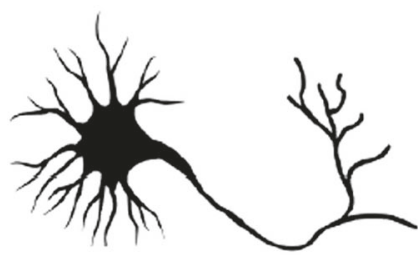

Derived from $S A D / F A D$ patients

Immune reaction

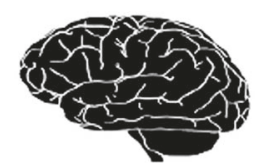

Derived from $S A D / F A D$ patients

Reproduce $A \beta$ pathology

(Plaques, oligomers)

(microglial cells)

Cognitive impairment

Features of $A \beta$ and Tau pathology

Genuine background

Screening of sporadic phenotype 'case by case'

Complete $A \beta$ and Tau pathology with neuronal degeneration

3D features:

- Spreading

- Organized neuronal population

- Complex cell interactions

\section{Limitations}

No neurofibrillary tangles

(Unless Tau mutations)

Accelerated phenotype

(Low contribution of aging)

No sporadic pathology
2D limitations:

- No plaques or tangles,

- Limited cell interactions

- Altered transcriptomic

Lack of immune cells
Immature cells

(limited vascularization)

No microglial cells

Rare oligodendrocytes

Pre-natal brain

transcriptomic profile

Low synaptic activity
Fig. 1 Features and limitations of humanized Alzheimer's disease models. Humanized animal models have the advantage of covering multiple aspects of the pathology (including brain inflammation, metabolic alterations, or cognitive impairment) but are built with an artificial etiology and lack the specificity of the human genetic and molecular background. 2D iPSC neurons culture have a more relevant background and allow the study of unique features from sporadic cases, but the $2 \mathrm{D}$ system fail to reproduce complex brain cell to cell interactions which affect their transcriptomic profile and maturation. $3 \mathrm{D}$ brain organoids keep the same background advantages of the $2 \mathrm{D}$ systems while featuring complex cell interactions and cell populations. They reproduce the AD hallmarks admirably: A $\beta$ deposits, Tau tangles, and neuronal degeneration. The most critical limitations to overcome are the lack of complete vascularization and incomplete immune system (presence of astrocytes but no microglial cells)

with endosome alterations which are a key early event of the disease [15-17]. A first attempt in developing a 3D cell culture model of $\mathrm{AD}$ was accomplished using human neuronal progenitor cells genetically engineered to overexpress mutant APP and PS1 [18]. This approach resulted in increased extra-cellular desposits of the $A \beta$ peptide toxic specie 1-42 and Tau phosphorylation into detergentinsoluble fibrils. Here, Gonzalez and coworkers, moved forward to develop a more physiological model of AD brain organoids generated from iPSC of FAD and Down syndrome patients [8]. Through an in vitro developmental process, $\mathrm{AD}$ brain organoids showed a progressive peptide accumulation and Tau phosphorylation, combined 
accumulation of $\mathrm{A} \beta$ peptide with amyloidogenic properties, in addition to accumulate plaque-like structures, preceding the appearance of phosphorylated Tau and neurofibrillary tangles. Measurements of caspase- 3 activation also indicated neuronal death in the $\mathrm{AD}$ brain organoids. The experimental model was highly reproducible and characterized on a quantitative manner.

Overall, the results obtained with $\mathrm{AD}$ brain organoids further support the close relationship between $\mathrm{A} \beta$ deposition and Tau phosphorylation. The use of AD minibrains will open the possibility of validating disease mechanisms and hopefully identify new pathways contributing to $\mathrm{AD}$ progression. For example, the possible role of the glycogen synthase kinase $3 \beta$ (GSK3 $\beta$ ) in Tau phosphorylation could be further explored, since $2 \mathrm{D}$ and $3 \mathrm{D}$ human $\mathrm{PPSC} A D$ cultures resulted in A $\beta$, Tau, and GSK-3 $\beta$ activity $[15,18]$. Among other molecular features linked to $\mathrm{AD}$, endosomal and lysosomal alterations with altered expression of Rab5 were reported in brain organoids derived from FAD stem cells [19]. Some SAD and FAD iPSC 2D systems also reported endosomal and lysosomal alterations, along with increased expression of endoplasmic reticulum (ER) stress markers [16, 17, 20]. ER stress is proposed as a key feature of $\mathrm{AD}$ and others neurodegenerative disease [21, 22]. However, there is inconsistency in the identification of ER stress markers using AD transgenic mice [23, 24]. Thus, human brain organoids could open new possibilities to identify the regulatory network affected in $\mathrm{AD}$ brains and define strategies for their intervention. The spreading of protein misfolding by a Prion-like mechanism is also emerging as a relevant factor mediating disease progression in $\mathrm{AD}$, a phenomenon that could be also studied and intervened using the human mini-brain technology [25].

Considering the demonstrated low translational potential of rodent models of $\mathrm{AD}$, humanized models should be considered as a complementary platform for drug screening to support preclinical studies. Results in iPSC-derived neuronal cultures from FAD patients showed important variations compared to non-human models in the dose required to obtain significant activity of $\gamma$-secretase modulators or inhibitors [26]. In addition, undirect effects of $\beta$ secretases inhibitors on Tau protein phosphorylation were also observed [15, 27]. Studies using pharmacological modulators of the $\beta$-secretase or $\gamma$-secretase yielded convincing results in $\mathrm{AD}$ brain organoids, with reductions of $\mathrm{A} \beta$ peptide levels and also Tau pathology, in agreement with previous results in iPSC cultures [18, 19]. In addition, the interest for gene-therapy approaches or stem-cell-based regenerative strategies for $\mathrm{AD}$ is increasing [28-30], which could be improved and complemented using AD minibrains. Clinical trials with gene therapy to deliver the nerve growth factor (NGF) [31] or the injection of genetically modified stem cells [32], showed overall good safety profiles. However, the therapeutic benefits obtained were limited despite the encouraging results observed in rodents and non-human primates [33,34], highlighting again the need of new models that closely resemble the human condition to study the efficacy of such interventions.

While brain organoids have great potential for future discovery initiatives, some technical limitations still need to be overcome. Aging is the main non-genetic risk factor to develop $\mathrm{AD}$, and the aging process is associated with numerous alteration including changes in gene expression [35]. However, neural cells derived from iPSC show a transcriptomic profile similar to prenatal brain [36]. In addition, the lack of vascularization in the brain organoid prevents further maturation of the neuronal cells, having adverse effects to the formation of active synapses while impairing the organoid survivability after prolonged periods of culture [11,37]. A possible solution to this issue may lie in the use of heterotypic cultures, with endothelial and mesenchymal stem cells [38]. Alternatively, chimeric models using the mouse brain vascularization system are under development [39]. Another factor limiting the use of brain organoid systems in the study of neurodegenerative disease is the lack of microglial cells, because they are not derived from the neural progenitor cell line [40]. However, microglial cells derived from human iPSC have been successfully integrated into 3D brain organoids structures, and appeared to be active and able to respond to localized injury [41]. In addition, a 3D culture model including neurons, astrocytes and microglia have been recently developped to modelize AD [42]. Thus, all these technological advances could be applied to the model developed by Soto's group to further resemble and mimic the pathological events observed in the AD brain. In addition, because hundreds of mini-brains can be generated at the same time, the possibility of developing drug screenings, or secondary validation of larger screenings using iPSC-derived AD neurons will accelerate the identification of compounds with therapeutic efficacy. Overall, 3D culture methods still needed to be refined, but certainly promise the implementation of clinically relevant models to accelerate the discovery of a cure or a treatment not only for $\mathrm{AD}$, but also for other major neurodegenerative diseases with high incidence such as Parkinson's disease. In this line of interest, a recent study used human mid-brain organoids to generate spatially organized clusters of dopaminergic neurons [43]. Finally, the possibility of studying the complex contributions of the genetic landscape of the human population in sporadic $\mathrm{AD}$ may be feasible in the future using brain organoids. The current study represents an inflection point for the discovery of hopefully effective approaches to treat AD.

Acknowledgements This study was supported by the FONDAP program 15150012, the US Office of Naval Research-Global (ONR-G) 
N62909-16-1-2003, the Millennium Institute P09-015-F, FONDEF ID16I10223, FONDEF D11E1007, the US Air Force Office of Scientific Research FA9550-16-1-0384, and CONICYT-Brazil 441921/ 2016-7.

\section{Compliance with ethical standards}

Conflict of interest The authors declare that they have no conflict of interest.

Publisher's note: Springer Nature remains neutral with regard to jurisdictional claims in published maps and institutional affiliations.

\section{References}

1. Jucker M. The benefits and limitations of animal models for translational research in neurodegenerative diseases. Nat Med. 2010;16:1210-4.

2. Zahs KR, Ashe KH. 'Too much good news' - are Alzheimer mouse models trying to tell us how to prevent, not cure, Alzheimer's disease? Trends Neurosci. 2010;33:381-9.

3. Serrano-Pozo A, Frosch MP, Masliah E, Hyan BT. Neuropathological alterations in Alzheimer disease. Cold Spring Harb Perspect Med. 2011;1:a006189.

4. Walsh DM, Selkoe DJ. A beta oligomers - a decade of discovery. J Neurochem. 2007;101:1172-84.

5. Sasaguri H, Nilsson P, Hashimoto S, Nagata K, Saito T, Strooper $\mathrm{BD}$, et al. APP mouse models for Alzheimer's disease preclinical studies. EMBO J. 2017;36:2473-87.

6. Andorfer C, Kress Y, Espinoza M, Silva RD, Tucker KL, Barde YA, et al. Hyperphosphorylation and aggregation of tau in mice expressing normal human tau isoforms. J Neurochem. 2003;86:582-90.

7. Kitazawa M, Medeiros R, LaFerla FM. Transgenic mouse models of Alzheimer disease: developing a better model as a tool for therapeutic interventions. Curr Pharm Des. 2012;18:1131-47.

8. Gonzalez C, Armijo E, Bravo-Alegria J, Becerra-Calixto A, Mays CE \& Soto C. Modeling amyloid beta and tau pathology in human cerebral organoids. Mol Psychiatry. 2018; https://doi.org/10.1038/ s41380-018-0229-8.

9. Tiscornia G, Vivas EL, Belmonte JCI. Diseases in a dish: modeling human genetic disorders using induced pluripotent cells. Nat Med. 2011;17:1570-6.

10. Lancaster MA, Renner M, Martin CA, Wenzel D, Bicknell LS, Hurles ME, et al. Cerebral organoids model human brain development and microcephaly. Nature. 2013;501:373-9.

11. Di Lullo E, Kriegstein AR. The use of brain organoids to investigate neural development and disease. Nat Rev Neurosci. 2017;18:573-84.

12. Mariani J, Coppola G, Zhang P, Abyzov A, Provini L, Tomasini L, et al. FOXG1-dependent dysregulation of GABA/glutamate neuron differentiation in autism spectrum disorders. Cell. 2015;162:375-90.

13. Birey F, Andersen J, Makinson C, Islam S, Wei W, Huber N, et al. Assembly of functionally integrated human forebrain spheroids. Nature. 2017;545:54-59.

14. Qian X, Nguyen HN, Song MM, Hadiono C, Ogden SC, Hammack $\mathrm{C}$, et al. Brain-region-specific organoids using mini-bioreactors for modeling ZIKV exposure. Cell. 2016;165:1238-54.

15. Israel MA, Yuan SH, Bardy C, Reyna SM, Mu Y, Herrera C, et al. Probing sporadic and familial Alzheimer's disease using induced pluripotent stem cells. Nature. 2012;482:216-20.

16. Muratore CR, Rice HC, Srikanth P, Callahan DG, Shin T, Benjamin LNP, et al. The familial Alzheimer's disease APPV717I mutation alters APP processing and Tau expression in iPSCderived neurons. Hum Mol Genet. 2014;23:3523-36.
17. Cataldo AM, Peterhoff CM, Troncoso JC, Gomez-Isla T, Hyman BT, Nixon RA, et al. Endocytic pathway abnormalities precede amyloid $\beta$ deposition in sporadic Alzheimer's disease and down syndrome: differential effects of APOE genotype and presenilin mutations. Am J Pathol. 2000;157:277-86.

18. Choi SH, Kim YH, Hebisch M, Sliwinski C, Lee S, D’Avanzo C, et al. A three-dimensional human neural cell culture model of Alzheimer's disease. Nature. 2014;515:274-8.

19. Raja WK, Mungenast AE, Lin YT, Ko T, Abdurrob F, Seo J, et al. Self-organizing 3D human neural tissue derived from induced pluripotent stem cells recapitulate Alzheimer's disease phenotypes. PLoS ONE. 2016;11:e0161969.

20. Kondo T, Asai M, Tsukita K, Kutoku Y, Ohsawa Y, Sunada Y, et al. Modeling Alzheimer's disease with iPSCs reveals stress phenotypes associated with intracellular $\mathrm{A} \beta$ and differential drug responsiveness. Cell Stem Cell. 2013;12:487-96.

21. Hetz C, Saxena S. ER stress and the unfolded protein response in neurodegeneration. Nat Rev Neurol. 2017;13:477-91.

22. Scheper W, Hoozemans JJM. The unfolded protein response in neurodegenerative diseases: a neuropathological perspective. Acta Neuropathol. 2015;130:315-31.

23. Hashimoto S, Saido TC. Critical review: involvement of endoplasmic reticulum stress in the aetiology of Alzheimer's disease. Open Biol. 2018;8:1098rsob.180024.

24. Lee JH, Won SM, Suh J, Son SJ, Moon GJ, Park UJ, et al. Induction of the unfolded protein response and cell death pathway in Alzheimer's disease, but not in aged Tg2576 mice. Exp Mol Med. 2010;42:386-94.

25. Soto C. Transmissible protein: expanding the Prion Heresy. Cell . 2012;148:968-77.

26. Liu Q, Waltz S, Woodruff G, Ouyang J, Israel MA, Herrera C, et al. Effect of potent $\gamma$-secretase modulator in human neurons derived from multiple presenilin 1-induced pluripotent stem cell mutant carriers. JAMA Neurol. 2014;71:1481-9.

27. Moore S, Evans LDB, Andersson T, Portelius E, Smith J, Dias $\mathrm{TB}$, et al. APP metabolism regulates tau proteostasis in human cerebral cortex neurons. Cell Rep. 2015;11:689-96.

28. Combs B, Kneynsberg A, Kanaan NM. Gene therapy models of Alzheimer's disease and other dementias. Methods Mol Biol. 2016;1382:339-66.

29. Hunsberger JG, Rao M, Kurtzberg J, Bulte JWM, Atala A, LaFerla FM, et al. Accelerating stem cell trials for Alzheimer's disease. Lancet Neurol. 2016;15:219-30.

30. Sproul AA. Being human: The role of pluripotent stem cells in regenerative medicine and humanizing Alzheimer's disease models. Mol Asp Med. 2015;43-44:54-65.

31. Rafii MS, Tuszynski MH, Thomas RG, Barba D, Brewer JB, Rissman RA et al. Adeno-associated viral vector (serotype 2)nerve growth factor for patients with Alzheimer disease: a randomized clinical trial. JAMA Neurol. 2018; https://doi.org/10. 1001/jamaneurol.2018.0233

32. Tuszynski MH, Thal L, Pay M, Salmon DP, U HS, Bakay R, et al. A phase 1 clinical trial of nerve growth factor gene therapy for Alzheimer disease. Nat Med. 2005;11:551-5.

33. Bishop KM, Hofer EK, Mehta A, Ramirez A, Sun L, Tuszynski $\mathrm{MH}$, et al. Therapeutic potential of CERE-110 (AAV2-NGF): targeted, stable, and sustained NGF delivery and trophic activity on rodent basal forebrain cholinergic neurons. Exp Neurol. 2008;211:574-84.

34. Nagahara AH, Bernot T, Moseanko R, Brignolo L, Blesch A, Conner JM, et al. Long-term reversal of cholinergic neuronal decline in aged non-human primates by lentiviral NGF gene delivery. Exp Neurol. 2009;215:153-9.

35. López-Otín C, Blasco MA, Partridge L, Serrano M, Kroemer G. The hallmarks of aging. Cell. 2013;153:1194-217. 
36. Camp JG, Badsha F, Florio M, Kanton S, Gerber T, WilschBrauniger $\mathrm{M}$, et al. Human cerebral organoids recapitulate gene expression programs of fetal neocortex development. Proc Natl Acad Sci USA. 2015;112:15672-7.

37. Lancaster MA, Knoblich JA. Generation of cerebral organoids from human pluripotent stem cells. Nat Protoc. 2014;9:2329-40.

38. Takebe T, Enomura M, Yoshizawa E, Kimura M, Koike H, Ueno $\mathrm{Y}$, et al. Vascularized and complex organ buds from diverse tissues via mesenchymal cell-driven condensation. Cell Stem Cell. 2015;16:556-65.

39. Mansour AA, Gonçalves JT, Bloyd CW, Li H, Fernandes S, Quang D, et al. An in vivo model of functional and vascularized human brain organoids. Nat Biotechnol. 2018;36:432-41.
40. Ginhoux F, Greter M, Leboeuf M, Nandi S, See P, Gokhan S, et al. Fate mapping analysis reveals that adult microglia derive from primitive macrophages. Science. 2010;330:841-5.

41. Muffat J, Li Y, Yuan B, Mitalipova M, Omer A, Corcoran S, et al. Efficient derivation of microglia-like cells from human pluripotent stem cells. Nat Med. 2016;22:1358-67.

42. Park J, Wetzel I, Marriott I, Dréau D, D’ Avanzo A, Kim YD, et al. 3D human triculture system modeling neurodegeneration and neuroinflammation in Alzheimer's disease. Nature Neurosci. 2018;21:941-51.

43. Monzel AS, Smits LM, Hemmer K, Hachi S, Moreno EL, Wuellen T, et al. Derivation of human midbrain-specific organoids from neuroepithelial stem cells. Stem Cell Rep. 2017;8:1144-54. 